$$
\begin{aligned}
& -1-7 \\
& \text { CCNF-89CRULL- }
\end{aligned}
$$

\title{
ULTRA-RELATIVISTIC HEAVY ION COLLISIONS IN A MULTI-STRING MODEL
}

\author{
K. Werner \\ Physics Department \\ BNL- -40369 \\ Brookhaven National Laboratory \\ Upton NY 11973 \\ DE8 8002123
}

\begin{abstract}
We present a model for ultra-relativistic heavy ion collisions based on color string formation and subsequent independent string fragmentation. Strings are formed due to color exchange between quarks at each individual nucleon nucleon collision. The fragmentation is treated as in $e^{+} e^{-}$or lepton nucleon scattering. Calculation for $p p, p A$, and $A A$ were carried out using the Monte Carlo code VENUS for Very Energetic NUclear Scattering (version 1.0).
\end{abstract}

\author{
Invited talk \\ Sixth International Conference on \\ Ultrarelativistic Nucleus-Nucleus Collisions \\ QUARK MATTER 1987 \\ Schloss Nordkirchen, West Germany \\ August 24-28, 1987
}

Die Arbeit wurde mit Unterstützung eines Stipendiums des Wissenschaftsausschusses der NATO über den DAAD ermöglicht.

This manuscript has been authored under contract number DE-AC02-76CH00016 with the U.S. Department of Energy. Accordingly, the U.S. Government retains a non-exclusive, royalty-free license to publish or reproduce the published form of this contribution, or allow others to do so, for U.S. Government purposes.

\section{DISCLAIMER}




\section{Introduction}

Currently at CERN and Brookhaven, experiments with heavy ions are performed with the aim of finding a new kind of matter: the Quark Gluon Plasma (QGP) ${ }^{1,2}$. In order to detect such a new phenomenon it is first of all absolutely necessary to understand the "normal" event, which is expected to occur for colliding nuclei not energetic or heavy enough to provide a phase transition.

In order to understand such "normal" collisions, extrapolations of successful proton proton models (like the Dual Parton Model (DPM) ${ }^{3-7}$ or the Lund model ${ }^{8,9}$ ) have been introduced. Since such an extrapolation is not unique, experimental assistance is needed to determine these models completely. Fortunately for energies not high enough and (or) nuclei not heavy enough, no plasma is expected, and in this case the models should be able to reproduce the data. Once a model is reliable in this sense, a severe deviation at larger values of the critical parameters, energy and mass, strongly indicates at least "something new."

In this paper we present a model for $p p, p A$, and $A A$ collisions which is based upon the assumption that string formation and fragmentation occur independently, and that all the strings fragment independently of each other. The last assumption is of course questionable for heavy nuclei because in this case many strings overlap. As in all DPModels, string formation is assumed to be a consequence of color exchange between quarks, so a string is stretched between a projectile parton and a target parton. This is very different in the Lund model, where a string is just an excited projectile or target nucleon. So, whereas the Lund model requires an empirical excitation function to determine the strings, the string properties in DPModels can be derived from the partonic structure of the nucleon (plus factorization assumptions in the case of multiple collisions). The model presented here is more general than the classical DPMs, taking into account more contributions. So, for example, the first color exchange does not only involve valence quarks but also sea quarks (the structure functions determins the ratio) which has important consequences. Another difference is the possibility of diquark breakup after two color exchanges. 
In chapter 2 we will describe the $p p$ model and compare results with data; in chapter 3 we discuss the extrapolation towards $A A$ and show some first results.

\section{Proton proton collisions}

Color exchange is assumed to cause the formation of color strings: $i$ exchanges of color between quarks of the projectile and target proton result in $2 i$ strings. The relative weight of $i$ color exchanges is named $w_{i}$, thus cross sections can be written as

$$
\sigma=\sum_{i=1}^{\infty} w_{i} \sigma_{i}
$$

$\sigma_{i}$ being the contribution consisting of $2 i$ strings.

We first describe the dominant $i=1$ contribution, i.e. the formation of two strings. Color exchange between a quark of the projectile and a quark of the target rearranges the color structure of the pp system; instead of two protons in singlet states we find two singlets each consisting of a diquark and a quark of the other nucleon (see fig. 1(a)). We explicitly treat the case in which one (or both) of the quarks participating in the color exchange is accompanied by an antiquark such that the $q \bar{q}$ pair is color neutral, because in this case the diquark quark $(q q-q)$ string is replaced by a $q-\bar{q}$ string and a baryon. In figs. $1(\mathrm{~b}, \mathrm{c}, \mathrm{d})$ we show this for the case when the projectile quark (b), the target quark (c), or both quarks (d) are part of colorless $q \bar{q}$ pairs. We generate quarks with and without $\bar{q}$ partners with probabilities $w$ and $1-w$, so the relative weights of the contributions $1(\mathrm{a}, \mathrm{b}, \mathrm{c}, \mathrm{d})$ can be expressed in terms of the parameter $w$ (in a complicated way because certain events have to be discarded as unphysical). So far we treat neither color exchange between antiquarks nor color exchange between gluons. Gluons are only spectators, in the sense that diquarks are implicitly assumed to be "dressed", i.e. to contain gluons.

Putting together two or more of the contributions $1(\mathrm{a}-\mathrm{d})$ provides higher order graphs: the color exchange between quarks being part of white $q \bar{q}$ pairs $1(\mathrm{~d})$ may occur in addition to the nondiffractive color exchange of fig. 1(a) leading to an $i=2$ (2 color exchanges) contribution with $2 i=4$ strings. Two color exchanges of the type $1(\mathrm{~d})$ in addition to the exchange of type 1 (a) leads to a $i=3$ contribution with six strings. Another possibility involving two-color exchanges is applying two times the exchange of type 1 (a) leading to a 
fast (!) forward-moving projectile quark coupled to a slow backward-moving target diquark (plus a corresponding string moving opposite).

The entire contribution $\sigma_{i}$ consists of all possibilities involving $i$ color exchanges between projectile and target quarks with the restrictions that each quark contributes only once to a color exchange and that the number of excess quarks (over antiquarks) does not fall below $N_{0} . N_{0}=2$ means: the diquark survives (this is assumed in other DPMs), $N_{0}=1$ allows a breakup of the diquark as described above (this is the value we use).

In order to determine string properties, like for example flavor content, rapidity and mass distribution of strings, we use the knowledge about the partonic structure of the nucleoul ${ }^{10}$. We assume that quarks taking part in color exchange have the same longitudinal momentum distribtuion as the quarks scattered in deep inelastic lepton nucleon collisions. A justification of this extrapolation from large $Q^{2}$ results toward small $Q^{2}$ is the following: the general form of the distribution functions may very well change by going to smaller $Q^{2}$, but the (very important) form of the divergences $x^{-\alpha}$ for small $x$ should survive. For the $p_{t}$ distribution of the quarks we make an exponential ansatz with a free parameter $\left\langle p_{t}\right\rangle$, which should be of the order (proton size) ${ }^{-1}$. With the assumption of massless quarks, antiquarks, and diquarks, the strings are completely determined.

As described in Ref. 10, we use a Feynman Field type procedure ${ }^{20}$ to fragment the strings, which have been formed according to the above prescription. Strings are treated as two independent parton jets, each parton jet producing successively particles according to elementary vertices. Such a vertex describes the flavor flow of a parton branching into a hadron and a remainder parton, where the latter one continues to branch again - if the energy is sufficiently high. Figure 2 shows the vertices we take into account: (a) a quark jet producing a meson, leaving a quark jet; (b) a quark jet producing a baryon, leaving an antidiquark jet; (c) a diquark jet producing a meson, leaving a diquark jet; and (d) a diquark jet producing a baryon, leaving an antiquark jet (vertices for antiquark and antidiquark jets are obtained by exchanging quarks and antiquarks).

The energy of a primary hadron relative to the energy of the corresponding jet is generated according to so-called splitting functions $f_{q}^{m}(x), f_{q}^{b}(x), f_{q q}^{m}(x)$, und $f_{q q}^{b}(x)$ for the four vertices of fig. 2. For reasons explained in Ref. 10 we use (up to normalization 
factors)

$$
\begin{aligned}
f_{q}^{m}(x) & =(1-x) \\
f_{q}^{b}(x) & =x^{\alpha}(1-x)^{3} \\
f_{q q}^{m}(x) & =(1-x)^{3} \\
f_{q q}^{b}(x) & =x^{\alpha}(1-x)
\end{aligned}
$$

As for the quarks inside the nucleons, we generate transverse momentum $\vec{p}_{t}$ for the quarks produced during fragmentation according to an exponential distribution, using the same parameter $\left\langle p_{t}\right\rangle$ (which is certainly not necessary, but both $\left\langle p_{t}\right\rangle$ should be of the same order). The antiquark assumes $-\overrightarrow{p_{t}}$.

All the parameters determining the fragmentation are fixed by comparing with $e^{+} e^{-}$ and deep inelastic scattering data ${ }^{10}$. The momentum transfer $Q^{2}$ entering the structure function is taken to be $Q^{2}=4 \mathrm{GeV}^{2}$. The probability $w$, that in a $p p$ collision an interacting quark is accompanied by an antiquark, is fixed such that the fraction of events with one surviving proton matched the ratio $\sigma_{\text {difr }} / \sigma_{\text {inel, }}$, which has, over a wide energy range, the value $0.2^{11}$. This prescription is explained in Ref. 12. For the mean transverse momentum $\left\langle p_{t}\right\rangle$ of quarks in the proton, we use $\left\langle p_{t}\right\rangle=0.45 \mathrm{GeV}$. For the multicolor exchange probabilities, $w_{i}$ entering eq. 1 , we use an exponential distribution

$$
w_{i}=\frac{1}{\langle i\rangle}\left(\frac{\langle i\rangle-1}{\langle i\rangle}\right)^{i-1}
$$

with energy independent mean value $\langle i\rangle=1.65$. It is obvious from this discussion of parameters that $p p$ collisions are essentially (with very few additional parameters) expressed in terms of string fragmentation.

In the following we compare Monte Carlo results (VENUS 1.0) for proton proton collisions with data. Figure 3 shows multiplicity distributions at $\sqrt{s}=23$ and $53 \mathrm{GeV}$ compared to the KNO function $\psi(n /<n>)$ of Ref. 13 which provides a (energy independent!) parametrization of data in a wide energy range. Surprisingly, the Monte Carlo results match the KNO curve perfectly, although (different to other models!) there are no free parameters fixed to enforce this agreement. The only parameter in this context, the mean number $\langle i\rangle$ of color exchanges, assumes the energy independent value of 1.65 in order to give the correct mean multiplicity at $\sqrt{s}=13.8 \mathrm{GeV}$. This automatically leads 
to correct mean multiplicities $\langle n\rangle$ for the whole ISR energy range and also to a correct description of multiplicity distributions at these energies (scaling)! Different to other DPModels, the wide multiplicity distributions are not due to multicolor exchanges (setting $<i>=1$ changes only very weakly $P(n))$. The broad distributions are due to the fact that not only diquark - valence quark strings contribute but also diquark - sea quark strings (with increasing weight at higher energies). Strings involving sea quarks lead to wider multiplicity distributions than strings with valence quarks (because of $q(x) \sim x^{-1}$ for sea quarks compared to $q(x) \sim x^{-1 / 2}$ for valence quarks at small $x$ ).

In figure 4 we compare $p_{t}$ distributions of $\pi^{+}$in the central rapidity region. Since most of the particles are produced in this region, this distribution is strongly correlated to $E_{t}$ distribution in nucleus nucleus collisions: a change in the mean value $\left\langle p_{t}(\pi)\right\rangle$ by a factor $\alpha$ results in a change of the $E_{t}$ scale by the same factor. Since, therefore, a few percent deviation for $\left\langle p_{t}(\pi)\right\rangle$ can result in very large deviation for the $E_{l}$ distribution, a very careful study of proton proton $p_{t}$ distribution has to be done. The input parameter determining the slope of the $p_{t}(\pi)$ distribution is $\left\langle p_{t}\right\rangle$, which governs the $p_{t}$ distribution of partons in nucleons as well as the $p_{t}$ distribution of $q-\bar{q}$ pairs during the fragmentation process. A value of $\left\langle p_{t}\right\rangle=0.45$ (as mentioned already) yields the VENUS results shown in fig. 4. The calculated mean value is $\left\langle p_{t}\left(\pi^{+}\right)\right\rangle=0.32 \mathrm{GeV}$ compared to the theoretical value of $0.33 \mathrm{GeV}$. The model (at least so far) does not include hard or semihard scattering contributions, which affect the tails of $p_{t}$ distributions.

\section{Nucleus Nucleus Collisions}

We try to make a straightforward extrapolation of the $p p$ model described in section 2 toward nucleus nucleus collisions without using new parameters. Since we introduced already the concept of multicolor exchange, it is very easy to generalize to collisions involving several projectile and target nucleons. Let us consider a sequence of collisions of projectile nucleons $n_{\alpha}$ with target nucleons $m_{\alpha}$, with not necessarily different nucleons $n_{\alpha}\left(m_{\alpha}\right)$. The first collision between the nucleons $n_{1}$ and $m_{1}$ performs as an ordinary $p p$ collision (compare chapter 2 ): $i_{1}$ color exchanges rearrange the singlet structure resulting in $2 i_{1}$ color strings. The number $i_{1}$ is generated according to the probability distribution $w_{i_{1}}$ of Eq. (3). A new situation occurs when in a later collision nucleons are involved which 
have already suffered a collision. In this case, we have to consider collisions between strings rather than nucleons, but this does not pose any difficulty: the collision $\alpha$ between the strings $n_{\alpha}$ and $m_{\alpha}$ consists (as for a $p p$ collision) of $i_{\alpha}$ color exchanges between quarks of string $n_{\alpha}$ and string $m_{\alpha}$, where only quarks participate which did not collide earlier. So, if for example a string consists of a fast-moving diquark and a quark moving opposite, only the diquark ( + sea) contributes to color exchange.

A problem arises whenever more than one color exchange occurs for one nucleon, because then a multi-parton distribution function $q_{i_{1} i_{2} \ldots i_{1}}\left(x_{1} x_{2} \ldots x_{n}\right)$ is required rather than the simple inclusive distribution function $q_{i}(x)$. For simplicity we make a factorization ansatz

$$
q_{i_{1} i_{2} \ldots i_{\ominus}}\left(x_{1} x_{2} \ldots x_{\lrcorner}\right)=\prod q_{i_{1}}\left(x_{1}\right) q_{i_{2}}\left(x_{2}\right) \ldots q_{i_{d}}\left(x_{J}\right) \Theta\left(1-\Sigma x_{i}\right) .
$$

To determine the sequence of $N$ collisions $n_{1} m_{1}, n_{2} m_{2} \ldots n_{N} m_{N}$ we forget about strings and use a purely geometrical picture of two nuclei consisting of $N_{A}$ and $N_{B}$ nucleons, distributed according to a Wood-Saxon desisity distribution. After shifting nucleus $A$ by an impact parameter $\vec{b}=\left(b_{x}, b_{y}\right)$, the projection of the nucleons to the $x-y$ plane defines collisions whenever two nucleons come closer than $\sqrt{\sigma / \pi}$. For $p . A$ collisions, this procedure amounts to the same as using the Glauber formula ${ }^{14}$

$$
\sigma_{i}=\int d^{2} b \frac{T(b)}{\nu !} e^{-T(b)}
$$

with a mean number of collisions $T(b)$ at given impact parameter $b$ :

$$
T(b):=\sigma_{\text {in }} \int d z \rho(z, b) \text {. }
$$

Since (as promised) no further parameter occurred compared to $p p$, and since the (few) $p p$ parameters are already adjusted, we have no further freedom and can proceed straight on to calculate $p A$ and $A A$ observables. Of course, this statement is not entirely true, since, for example, the factorization assumption Eq. (4) provides some kind of parameter which has to be tested and eventually modified by comparison with data.

In the following we want to discuss very first VENUS results for $p A$ and $A A$ at 200 $A G e V$. Figure 5 demonstrates that the model (VENUS) reproduces the negative particle multiplicity distributions of Ref. 15 not only for $p p$ but also for $p A r$ and $p X e$. In the same way the charged particle multiplicity distributions can be reproduced (no figure). 
We obtain for the mean charged multiplicities $<n_{c h}>$ the values $7.7,12.6$, and 16.7 in agreement to the experimental values of 7.713 .3 , and 17.3 and in rough agreement to the empirical law

$$
<n(\bar{\nu})>=0.5<n(1)>(\bar{\nu}+1)
$$

since the mean number of collisions $\bar{\nu}$ assumes the values 2.1 and 3.1 for $p A r$ and $p X e$. $\mathrm{Eq} .(7)$ is a consequence of the wounded nucleon model ${ }^{16}$, where $\nu$ collisions correspond to $\nu+1$ sources of particle production ("excited nucleons"), especially a $p p$ collision $(\nu=1)$ consists of two sources. The reason that this simple law is still approximately true although the situation is much more complicated is the following: due to multicolor exchange, the $q-\bar{q}$ strings are really higher order contributions in the sense that nearly all the particles are produced from quark diquark strings. On the other hand, independent of higher order effects, we have exactly $\nu+1$ such $q-q q$ strings for the case of $\nu$ collisions. So, we have approximately

$$
<n(\nu)>\approx<n(q-q q)>(\nu+1) \approx 0.5<n(1)>(\nu+1)
$$

in agreement with the observation (Eq. (7))

In figure 6 we display the distribution of the transverse energy

$$
E_{t}=\sum \sin \theta_{i} \cdot E_{i}
$$

including all particles in the central rapidity interval $2.2<y<3.8$. Since both data ${ }^{17}$ and Monte Carlo results (VENUS) are still preliminary, we don't want to conclude much from these results. It should be considered as a demonstration that the model, at least qualitatively, reproduces the data, showing a similar plateau behavior (as other models 9,18 do too).

\section{Conclusions}

We have introduced a string model for $p p$ collisions which very successfully describes inclusive and exclusive scattering data. The model can be extended in a (nearly) straightforward way toward nucleus nucleus collisions. From first comparison with data, we conclude (as other authors too 9,18 ) that at least some $p A$ and $A A$ data presently available 
can be described by $p p$ extrapolation. Yet, much more detailed comparisons are necessary to confirm this statement. An important future task is the question, where differences between the existing Monte Carlo models manifest themselves. There are more than only conceptual differences between the models: Lund $^{8,9}$ predicts, for example, increasing string masses with increasing collision number, which is just the opposite for DPModels. The model presented above has several striking differences to all other DPMs: the inclusion of sea quarks for $q-q q$ strings, for example, strongly affect multiplicity distribution, leading naturally to KNO type multiplicity distributions.

\section{Acknowledgements}

I gratefully acknowledge discussions with U. Heinz, S. Kahana, A. Matheson, M. Tannenbaum, J. Hüfner, and J. Aichelin. This work has been supported by the U. S. Department of Energy under contract no. DE-AC02-76CH00016.

Die Arbeit wurde mit Unterstützung eines Stipendiums des Wissenschaftsausschusses der NATO über den DAAD ermöglicht. 


\section{References}

1. Proceedings of this conference.

2. Proc. of the Fifth International Conference on Ultra-Relativistic Nucleus-Nucleus Collisions, Asilomar; eds. L. Schroeder and M. Gyulassy, Nucl. Phys. A461 (1987).

3. A. Capella and J. Tran Thanh Van, Z. Phys. C10, 249 (1981).

4. A. Capella, T. A. Casado, C. Pajares, A. V. Ramello, J. Tran Thanh Van, Z. Phys. C33, 541 (1987).

5. J. Ranft and S. Ritter, Z. Phys. C27, 413 (1985).

6. P. Aurenche, F. W. Bopp, J. Ranft, Z. Phys. C23, 67 (1984).

7. T. P. Pansart in (2).

8. B. Anderson, G. Gustafson, and B. Nielsson-Almqvist, Nucl. Phys. B281, 289 (1987).

9. M. Gyulassy, CERN-TH $4794 / 87$.

10. K. Werner, Preprint BNL-39726, to be published in Phys. Lett.B.

11. A. Wroblewski, in:Proc. of the 14th International Symposium on Multiparticle Dynamics, Granlibakkan, 1983; eds. P. Yager and G. F. Gunion, World Scientific Publ. Co.

12. K. Werner, M. Kutschera, Phys. Lett. 183E, 385 (198?).

13. P. Slattery, Phys. Rev. Lett. $\underline{29}, 1624$ (1972).

14. R. J. Glauber and G. Matthiae, Nucl. Phys. B21, 135 (1970).

15. C. DeMarzo et al, Phys. Rev. D26, 1019 (1982).

16. A. Bialas, M. Bleszynski, and W. Czyz, Nucl. Phys. B111, 461 (1976).

17. NA35; A. Bamberger et al, Phys. Lett. B184, 271 (1987).

18. J. Ranft, Phys. Lett. B188, 379 (1987).

19. B. Alper et al, Nucl. Phys. B100, 237 (1975).

20. R. D. Field, R. P. Feynman, Nucl. Phys. B136, 1 (1978). 


\section{Figure captions}

Figure 1: The four basic (one-color exchange) contributions to $p p$ collisions. Color exchange (arrow) is the basic mechanism to rearrange color singiets (closed lines) and thus to produce strings. The contributions a) to d) differ in the number $N$ of quarks being part of a white $q \bar{q}$ pair: $N=0$ for a), $N=1$ for b),c), and $N=2$ for d).

Figure 2: The elementary fragmentation vertices for the fragmentation of quark and diquark jets (antiquark and antidiquark fragmentation is obtained by exchanging quarks and antiquarks in the figure).

Figure 3: Multiplicity distributions for $p p$ collisions at $\sqrt{s}=23$ and $53 \mathrm{GeV}$, compared to the KNO function of Ref. 13

Figure 4: Transverse momentum distribution of $\pi^{+}$produced in $p p$ collisions at $\sqrt{s}=23 \mathrm{GeV}$ in the central rapidity region. The data are from Ref. 19.

Figure 5: Multiplicity distribution of negative particles produced in $200 \mathrm{GeV} p p, p A r$ and $p X e$ collisions. The data are from Ref. 15. The Argon results are multiplied by a factor of 10, the Xenon results by a factor of 100 .

Figure 6: Transverse energy $\left(E_{t}\right)$ distribution for a rapidity interval $2.2<y<3.8$ in a $200 \mathrm{AGeV}$ $O P b$ collision. The data are from Ref. 17 . 
a)

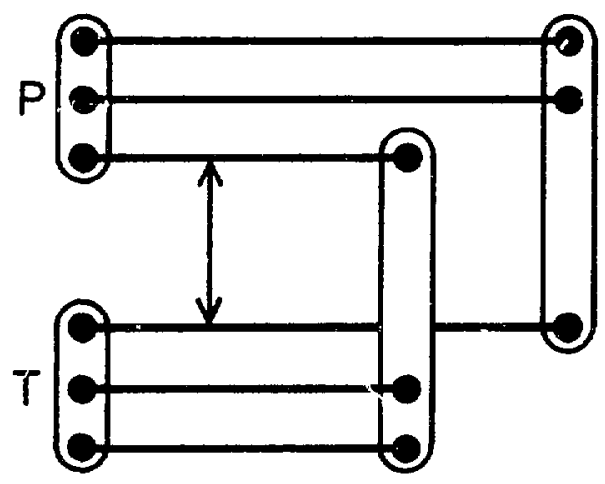

c)

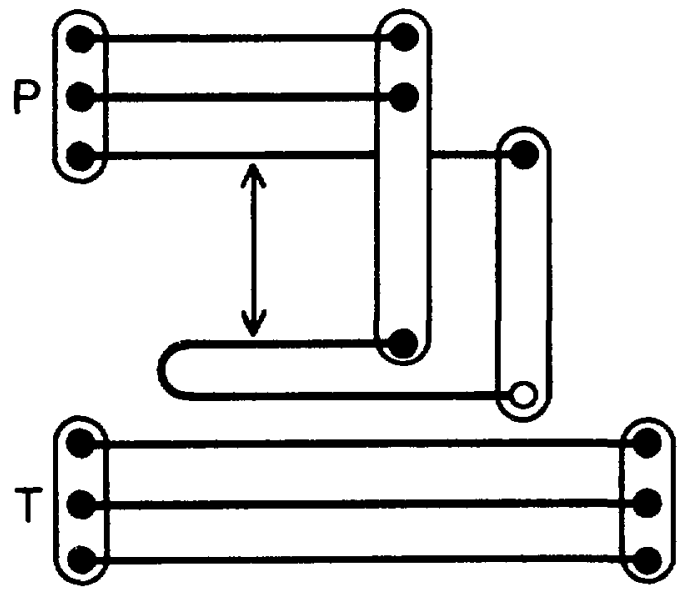

b)

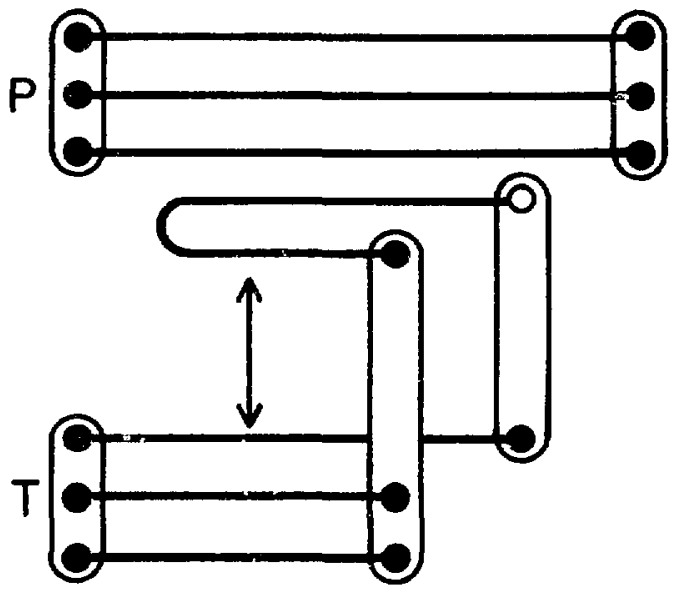

d)

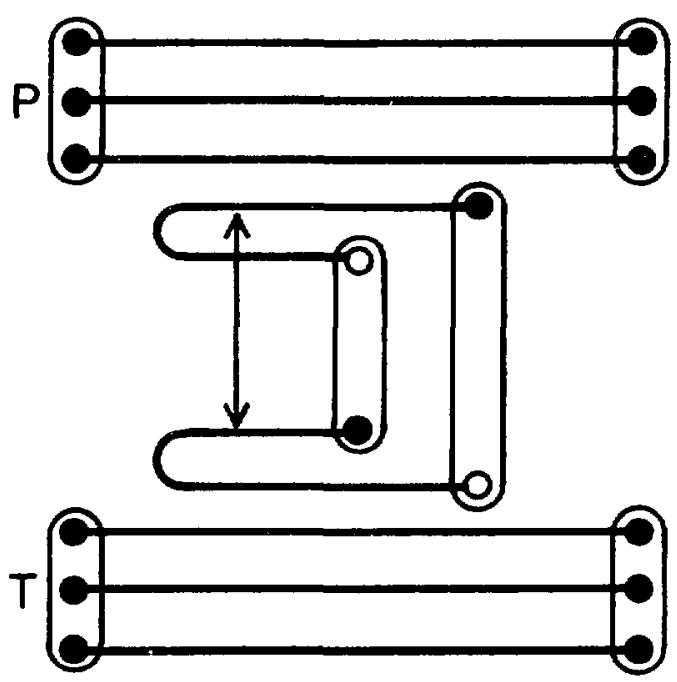

Figure 1 

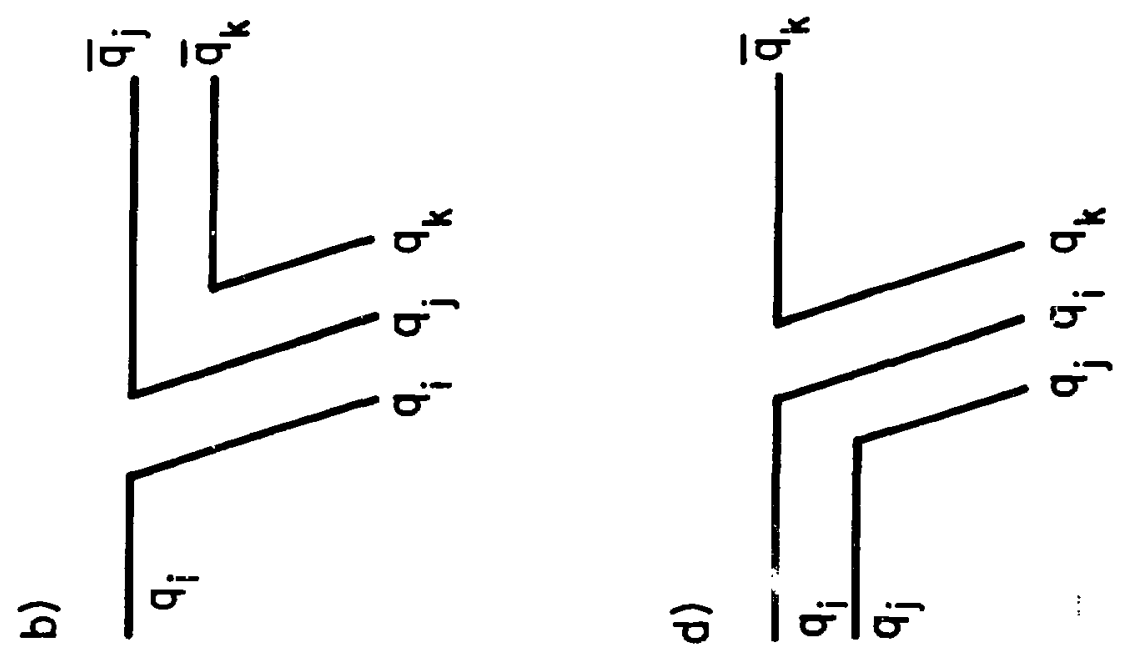

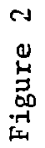
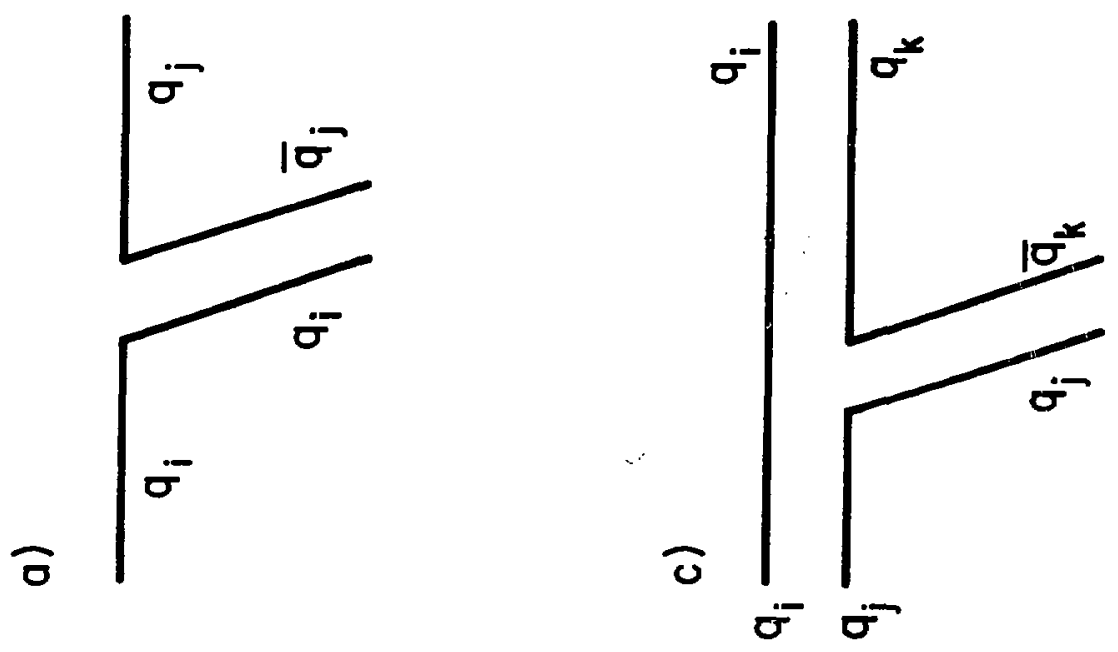

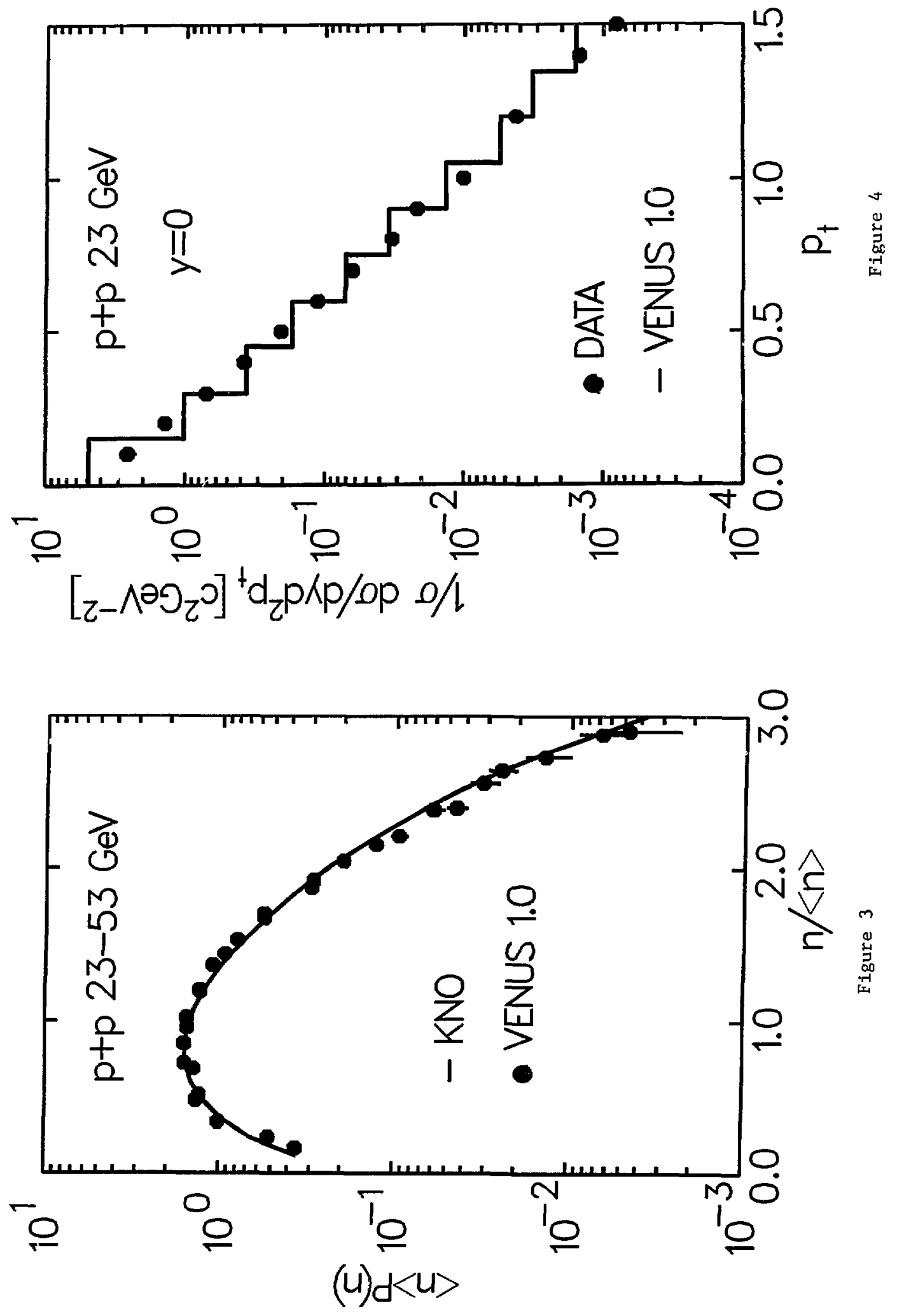




Figure 5

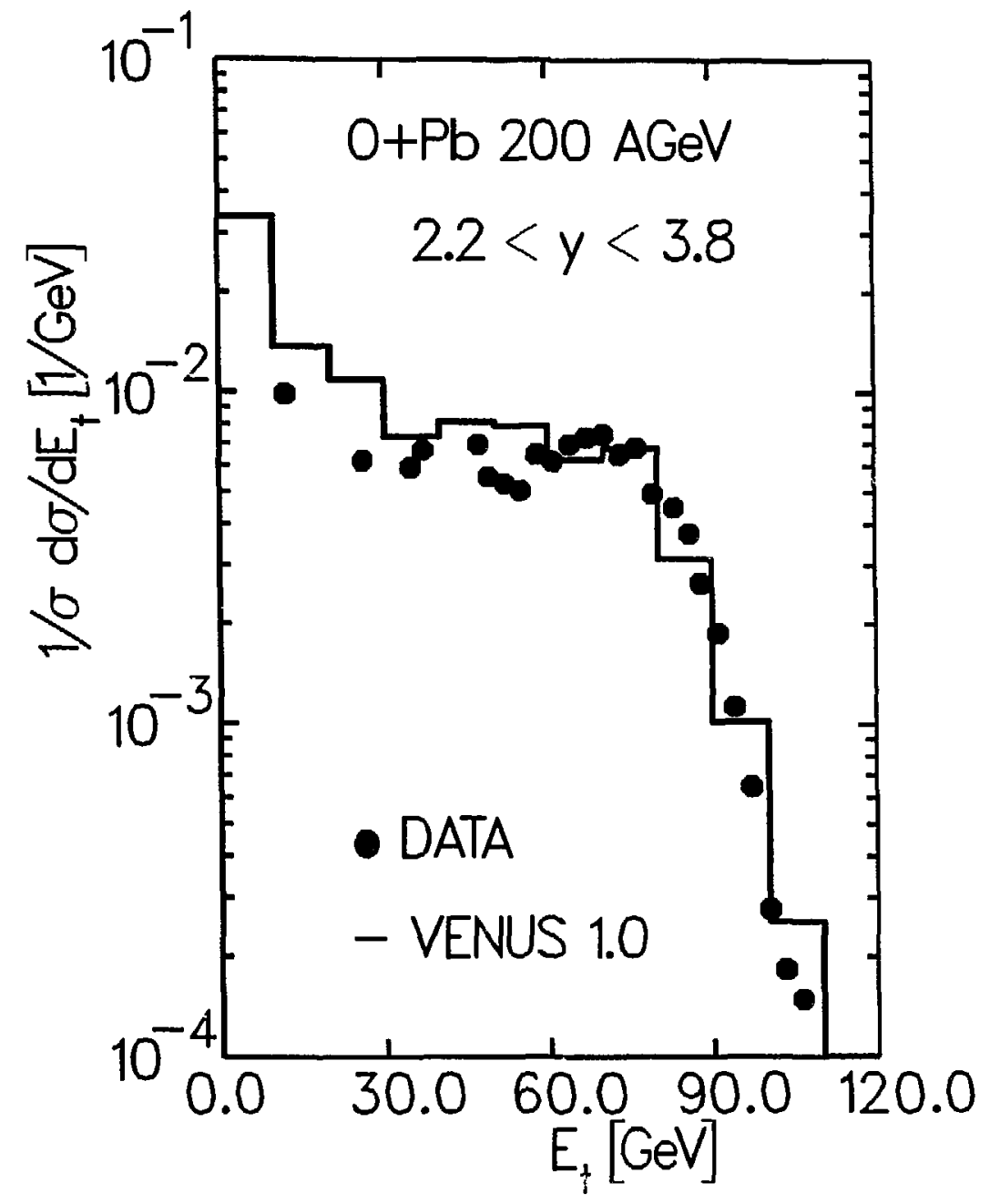

Figure 6 